\section{Transtorno da Compulsão Alimentar Periódica em Uma População de Obesos Mórbidos Candidatos a Cirurgia Bariátrica do Hospital Universitário Oswaldo Cruz, em Recife - PE}

\section{RESUMO}

Objetivos: Avaliar a freqüência de transtorno da compulsão alimentar periódica (TCAP) e dos principais transtornos psiquiátricos associados à obesidade mórbida em indivíduos à espera de cirurgia bariátrica. Métodos: Estudo de corte transversal. Foram entrevistados pacientes do programa de cirurgia da obesidade do Hospital Universitário Oswaldo Cruz e avaliados o perfil sócio-demográfico, a qualidade de vida (escala SF36), o TCAP (Binge Eating Scale - BES) e os transtornos psiquiátricos (Mini International Neuropsychiatry Interview - M.I.N.I./DSM-IV). Resultados: Dos 400 pacientes inscritos no programa, 67 (16,8\%) foram entrevistados. $\mathrm{O}$ IMC variou de 36,1 a $81,8 \mathrm{~kg} / \mathrm{m}^{2}$ (média $48,5 \pm 8,8$ ). Todos os entrevistados apresentavam doenças associadas, sendo a HAS, os distúrbios do sono e as osteopatias as mais freqüentes. Os transtornos psiquiátricos mais freqüentes foram: $47,8 \%$ transtorno de ansiedade generalizada; $29,9 \%$ depressão atual e $34,3 \%$ depressão no passado. Verificou-se TCAP em $56,7 \%$ dos pacientes (25,4\% TCAP moderado e 31,3\%, grave) e esses apresentaram os piores escores em todos os domínios de qualidade de vida da escala SF-36. Conclusões: Constatou-se elevada prevalência de TCAP. Grupo com TCAP apresentou maior número de tratamentos realizados com objetivo de perder peso, elevada prevalência de depressão maior no momento da avaliação, piores escores em todos os domínios da escala de qualidade de vida SF-36. Visto que os portadores de TCAP apresentam vasta psicopatologia e maior probabilidade de comprometimento nos resultados da cirurgia, deve-se aprimorar a detecção desses distúrbios a fim de proporcionar-lhes o tratamento adequado. (Arq Bras Endocrinol Metab 2006;50/5:901-908)

Descritores: Obesidade; Transtorno de compulsão alimentar periódica; Depressão; Qualidade de vida; Comorbidade

\begin{abstract}
Binge Eating Disorder in a Population of Morbid Obese Candidates to Bariatric Surgery at the Oswaldo Cruz University Hospital in Recife, PE.

Objectives: To analyze the frequency of binge eating disorder (BED) and of the main psychiatric disorders associated with morbid obesity in individuals on the waiting list for bariatric surgery. Method: Cross sectional study. Interviews with patients from the Surgery for Obesity Program of Oswaldo Cruz University Hospital were conducted evaluating sociodemographic profile, quality of life (SF-36 scale), BED (Binge Eating Scale - BES) and psychiatric disorders (M.I.N.I./DSM-IV). Results: 67 out of 400 patients enrolled in the program were interviewed (16.8\%). The BMl varied from 36.1 to $81.8 \mathrm{~kg} / \mathrm{m}^{2}$ (average $48.5 \pm 8.8$ ). All have associated diseases, the most frequent being systemic arterial hypertension, sleeping disorders and osteopathies. The most frequent psychiatric disorders were: 47.8\% generalized anxiety disorder, $29.9 \%$ major depressive disorder, single episode, $34.3 \%$ recurrent major depressive disorder. In this group $56.7 \%$ showed BED (25.4\% moderate and $31.3 \%$ severe) and the worse scores in all the domains of quality of life (SF-36 scale). Conclusions: High
\end{abstract}

artigo original

\author{
Kátia Petribu \\ Edilana Sá Ribeivo \\ Fabiana M.F. de Oliveiva \\ Cristiana I.A. Braz \\ Maria Laura Mota Gomes \\ Daniella E. de Araujo \\ Nair C.N. de Almeida \\ Pedro C. de Albuquerque \\ Moacir de N.L. Ferreira
}

Faculdade de Ciências Médicas, Universidade de Pernambuco (KP, ESR, FMFO, CIAB, MLMG \& DEA) e Hospital Universitário Oswaldo Cruz / HUOC (NCNA, PCA \& MNLF), Recife, PE.

Recebido em 29/09/05 Revisado em 07/02/06 e 28/04/06 Aceito em 30/04/06 
prevalence of BED. The compulsive eaters showed a higher number of obesity treatments, higher prevalence of actual major depression, and the worse scores in all the domains of the SF-36 scale. Considering the ample range of psychopathology associated with BED and the greater probability of jeopardizing the surgery results it is very important to improve the detection of these disorders in order to provide adequate treatment. (Arq Bras Endocrinol Metab 2006;50/5:901-908)

Keywords: Obesity; Binge eating disorder; Depression; Quality of life; Comorbidity

A OBESIDADE É UM IMPORTANTE problema de saúde pública, tendo em vista sua alta prevalência, a dificuldade no controle e o elevado índice de recidiva. Estima-se a freqüência de 5 a $30 \%$ do transtorno da compulsão alimentar periódica (TCAP) em obesos que buscam serviços especializados para tratamento da obesidade, embora tenha sido encontrada prevalência de até $50 \%$, comparada a $2-3 \%$ na população geral (1-4). No Brasil, Appolinário e cols. (5), Coutinho (6) e Borges (7) encontraram uma prevalência entre $15 \%$ e $22 \%$ em pacientes que procuravam tratamento para emagrecer. Entre os pacientes que realizaram a cirurgia bariátrica, esta prevalência pode variar de $27 \%$ a $47 \%(8,9)$. Aproximadamente $20 \%$ das pessoas que se identificam como portadoras de compulsão alimentar possuem diagnóstico de TCAP $(10,11)$. A dimensão variada encontrada na prevalência do TCAP é devida, em parte, às variações das definições de compulsão (11).

Binge eating (compulsão alimentar periódica) caracteriza-se pela ingestão de grande quantidade de comida em até duas horas, acompanhada da sensação de perda de controle sobre o que ou o quanto se come. Quando esses episódios ocorrem, pelo menos dois dias por semana nos últimos seis meses, associado a algumas características de perda de controle e não são acompanhados de comportamentos compensatórios dirigidos para a perda de peso, compõem a síndrome denominada binge eating disorder (TCAP/DSM IV-TR) (12).

Os obesos comedores compulsivos apresentam um início do quadro de obesidade mais precoce que os não-compulsivos, além de iniciarem mais cedo a preocupação com peso e dietas, desde as mais absurdas até as mais conservadoras. Eles apresentam ainda maior prevalência da flutuação de peso e passam mais tempo de suas vidas tentando emagrecer $(1,13)$.

O comer compulsivo representa uma dificuldade de tratamento dos pacientes obesos, pois indivíduos com esses episódios interrompem prematuramente os tratamentos pela incapacidade de controlar a ingestão alimentar. Esses indivíduos tendem a engordar se comparados àqueles sem esta perturbação. $O$ TCAP está relacionado a sintomas psicopatológicos em geral (sobretudo depressão), a uma maior gravidade da obesidade e prejuízo no funcionamento social e ocupacional $(6,10,13-16)$.

Indivíduos obesos da população geral ou que procuram tratamento para emagrecer não parecem evidenciar um aumento da morbidade psiquiátrica. Entretanto, ocorre um aumento da psicopatologia em pacientes gravemente obesos (obesidade grau III) $(17,18)$.

Diante da dificuldade em obter bons resultados nos tratamentos de redução de peso, a procura pela cirurgia da obesidade aumentou e vem sendo realizada com sucesso. Entretanto, existe sempre a preocupação de que alterações do comportamento alimentar possam vir a trazer complicações pós-operatórias e comprometer o resultado da cirurgia.

A avaliação psiquiátrica pré-operatória é fundamentalmente clínica, mas deve ser auxiliada utilizando-se testes psicológicos e entrevistas psiquiátricas estruturadas. Assim sendo, torna-se fundamental a avaliação psiquiátrica, mesmo que através de escalas de avaliação validadas e aplicadas pela equipe, dos pacientes candidatos à cirurgia bariátrica. $\mathrm{O}$ tratamento prévio do transtorno psiquiátrico associado à obesidade pode ser fundamental para o sucesso do procedimento cirúrgico.

Diante desses resultados, os objetivos deste estudo foram: 1) observar a prevalência de TCAP e dos principais transtornos psiquiátricos associados à obesidade mórbida em indivíduos à espera de cirurgia bariátrica; 2) determinar o perfil sócio-demográfico da população inscrita no programa de cirurgia da obesidade do HUOC-UPE; 3 ) avaliar a qualidade de vida e as comorbidades relacionadas com o comportamento alimentar desses indivíduos.

\section{MATERIAL E MÉTODOS}

\section{Sujeitos e Procedimentos}

Foram entrevistados 67 (16,75\%) dos 400 pacientes obesos mórbidos inscritos no programa de cirurgia da obesidade do Hospital Universitário Oswaldo Cruz (HUOC), Recife, PE, no período de agosto de 2004 a maio de 2005. A avaliação foi realizada no dia da reunião do grupo de obesos ou de suas consultas no ambulatório, por estudantes de medicina previamente treinadas para a aplicação dos instrumentos.

Arq Bras Endocrinol Metab vol 50 n 5 Outubro 2006 


\section{Materiais}

Os instrumentos utilizados na coleta de dados foram: 1- Questionário de perfil sociodemográfico e comorbidades associadas à obesidade mórbida

2- Mini International Neuropsychiatric Interview (M.I.N.I.) (19): O MINI é um questionário diagnóstico simples e breve destinado à pesquisa e mais abrangente que os instrumentos de triagem, constituindo-se numa alternativa mais econômica para a seleção de pacientes em ensaios clínicos, e pode também ser utilizado na prática clínica em psiquiatria. Foi desenvolvido por pesquisadores do Hospital PitiéSalpêtrière de Paris e da Universidade da Flórida. É um questionário breve (15-30 minutos), compatível com os critérios do DSM-III-R/IV e da CID-10 (versões distintas), que pode ser utilizado por clínicos após um treinamento rápido (de 1 a $3 \mathrm{~h}$ ). Compreende 19 módulos que exploram 17 transtornos do eixo I do DSM-IV, o risco de suicídio e o transtorno da personalidade anti-social.

3- Escala de Compulsão Alimentar Periódica [Binge Eating Scale - BES (20)]: A BES é um questionário auto-aplicável, adequado para discriminar indivíduos obesos de acordo com a gravidade da compulsão alimentar periódica (CAP). A escala é constituída por uma lista de 16 itens e 62 afirmativas, das quais deve ser selecionada, em cada item, aquela que melhor representa a resposta do indivíduo. A cada afirmativa corresponde um número de pontos de 0 a 3, abrangendo desde a ausência ("0") até a gravidade máxima ("3") da CAP. O escore final é o resultado da soma dos pontos de cada item. Indivíduos com pontuação menor ou igual a 17 são considerados sem compulsão; com pontuação entre 18 e 26 são considerados com compulsão moderada; e aqueles com pontuação maior ou igual a 27 , com a forma grave.

É válido como um instrumento de rastreamento. Sugere-se que seja utilizado o escore de 17 como ponto de corte para que se obtenha sensibilidade suficiente, e que o diagnóstico seja, sempre que possível, confirmado por uma entrevista clínica.

4- Escala de qualidade de vida SF-36 (21): A SF-36 é um instrumento genérico de avaliação da qualidade de vida, de fácil administração, compreensão e não extenso. É auto-administrável, multidimensional, formado por 36 itens englobados em oito escalas ou componentes: capacidade funcional, aspectos físicos, dor, estado geral de saúde, vitalidade, aspectos sociais, limitação por aspectos emocionais e saúde mental. Apresenta escore final de 0 a 100, onde zero corresponde a um pior estado geral de saúde e cem a um melhor estado.

\section{Procedimentos éticos}

O estudo foi previamente aprovado pelo Comitê de Ética em Pesquisa do HUOC/ UPE e todos os pacientes assinaram o termo de consentimento pósinformado. O estudo seguiu a orientação da declaração de Helsinki (1989) e da resolução n ${ }^{\circ}$ 196/96 sobre pesquisa envolvendo seres humanos do Conselho Nacional de Saúde.

\section{Análise estatística}

Foram utilizados o Microsoft Excel ${ }^{\circledR}$, o pacote estatístico Epi-info 6.2 e o software SAS (Statistical Analyis System) versão 8.0. O cálculo do tamanho amostral foi feito no módulo Analysis e Statcalc, devendo a entrevista ser realizada com apenas 16 pacientes, frente ao tamanho da população (400 pacientes), à freqüência esperada de TCAP (22\% em pacientes que procuravam tratamento para emagrecer) e ao pior resultado aceitável ( $2 \%$ na população geral). No entanto, foram entrevistados 67 pacientes, ou seja, 4,18 vezes o " $n$ " necessário ao nível de confiança de $95 \%$.

Para comparações entre variáveis nominais ou categóricas foram realizados testes não paramétricos (teste Qui quadrado ou teste exato de Fischer). Para as variáveis numéricas, o teste t de Student. O nível de significância considerado nas decisões dos testes estatísticos foi de $5 \%$.

\section{RESULTADOS}

Como pode ser verificado na tabela 1 , não houve diferença estatisticamente significante entre o grupo de obesos mórbidos com ou sem TCAP de acordo com as seguintes variáveis: sexo, idade, procedência, escolaridade, renda per capta, IMC atual e tempo médio de espera na lista. O tempo médio de espera foi de 2,23 anos. Predominaram o sexo feminino $(73,10 \%)$ e a cor parda $(43,30 \%)$. A idade variou de 19 a 57 anos (média de 42,10 $\pm 10,46$ anos). Com relação à instrução, $35,80 \%$ não concluíram o primeiro grau, $43,30 \%$ dos pacientes eram donas de casa e $58,70 \%$ detinham renda per capita familiar mensal inferior a um salário mínimo. O IMC dos pacientes variou de 36,06 a $81,79 \mathrm{~kg} / \mathrm{m}^{2}$ (média $48,51 \pm 8,75 \mathrm{~kg} / \mathrm{m}^{2}$ ). Todos os entrevistados apresentaram doenças associadas, sendo a HAS, os distúrbios do sono e as osteopatias as mais freqüentes. Já em relação ao número de tratamentos realizados com objetivo de perder peso, o grupo com TCAP tentou mais tratamentos do que o grupo sem TCAP, tendo sido essa diferença estatisticamente significante $(\mathrm{p}=0,005)$. 
Tabela 1. Distribuição das variáveis sócio-demográficas e clínicas segundo a presença ou não de TCAP.

\begin{tabular}{|c|c|c|c|c|}
\hline Variáveis & $\begin{array}{c}\text { Grupo com TCAP } \\
(n=38)\end{array}$ & $\begin{array}{c}\text { Grupo sem TCAP } \\
(n=29)\end{array}$ & $\begin{array}{l}\text { Grupo } \\
\text { total }\end{array}$ & $\mathbf{p}$ \\
\hline \multicolumn{5}{|l|}{ Sexo } \\
\hline Feminino & $30(78,9 \%)$ & $19(65,5 \%)$ & $49(73,1 \%)$ & $\mathrm{p}(1)=0,21$ \\
\hline Masculino & $8(21,1 \%)$ & $10(34,5 \%)$ & $18(26,9 \%)$ & \\
\hline Idade (anos; média \pm DP) & $43,2( \pm 10,2)$ & $40,7( \pm 10,8)$ & $42,1( \pm 10,5)$ & $p(2)=0,33$ \\
\hline \multicolumn{5}{|l|}{ Procedência } \\
\hline Grande Recife & $35(92,1 \%)$ & $24(82,8 \%)$ & $59(88,1 \%)$ & $p^{(1)}=0,28$ \\
\hline Interior/outro estado & $3(7,9 \%)$ & $5(17,2 \%)$ & $8(11,9 \%)$ & \\
\hline \multicolumn{5}{|l|}{ Escolaridade } \\
\hline $1^{\circ}$ grau incompleto & $18(47,4 \%)$ & $6(20,7 \%)$ & $24(35,8 \%)$ & $p(1)=0,16$ \\
\hline $1^{\circ}$ grau completo & $7(18,4 \%)$ & $8(27,6 \%)$ & $15(22,4 \%)$ & \\
\hline $2^{\circ}$ grau (in)completo & $9(23,7 \%)$ & $10(34,5 \%)$ & $19(28,4 \%)$ & \\
\hline \multirow{2}{*}{\multicolumn{5}{|c|}{ Renda per capita $\left(^{*}\right)$}} \\
\hline & & & & \\
\hline Menor que um & $22(61,1 \%)$ & $15(55,6 \%)$ & $37(58,7 \%)$ & $p^{(3)}=0,06$ \\
\hline Mais de um & $14(38,9 \%)$ & $12(44,4 \%)$ & $26(41,3 \%)$ & \\
\hline \multicolumn{5}{|l|}{$\begin{array}{l}\text { IMC atual (média } \pm \text { DP) } \\
N^{\circ} \text { de tratamentos clínicos }\end{array}$} \\
\hline Nenhum & $3(7,9 \%)$ & $2(6,9 \%)$ & $5(7,5 \%)$ & $p^{(3)}=0,005$ \\
\hline Um a 10 & $12(31,6 \%)$ & $18(62,1 \%)$ & $30(44,7 \%)$ & \\
\hline Mais de 10 & $23(60,5 \%)$ & $9(31,0 \%)$ & $32(47,8 \%)$ & \\
\hline $\begin{array}{l}\text { Tempo de espera na lista (anos; } \\
\text { média } \pm \text { DP) }\end{array}$ & $2,2( \pm 1,3)$ & $2,3( \pm 1,9)$ & $2,2( \pm 1,6)$ & $p^{(2)}=0,81$ \\
\hline
\end{tabular}

Tabela 2. Freqüência dos principais transtornos psiquiátricos explorados pelo M.I.N.I. segundo a presença ou não de TCAP.

\begin{tabular}{lcccc}
\hline Transtornos & Grupo com TCAP & Grupo sem TCAP & Grupo total & $\mathbf{p}$ \\
\hline Transtorno de ansiedade generalizada & $21(55,3 \%)$ & $11(37,9 \%)$ & $32(47,8 \%)$ & $\mathrm{p}(1)=0,15$ \\
Depressão atual & $16(42,1 \%)$ & $4(13,8 \%)$ & $20(29,9 \%)$ & $\mathrm{p}(1)=0,01$ \\
Depressão no passado & $11(28,9 \%)$ & $12(41,4 \%)$ & $23(34,3 \%)$ & $\mathrm{p}(2)=0,28$ \\
Agorafobia & $9(23,7 \%)$ & $5(17,2 \%)$ & $14(20,9 \%)$ & $\mathrm{p}(2)=0,52$ \\
Transtorno do pânico & $9(23,7 \%)$ & $2(6,9 \%)$ & $11(16,4 \%)$ & $\mathrm{p}(1)=0,09$ \\
\hline
\end{tabular}

(1) Teste Exato de Fisher; (2) Teste Qui-quadrado de Pearson

Na tabela 2 observa-se a distribuição dos principais transtornos psiquiátricos de acordo com o M.I.N.I., verificando-se em ambos os grupos uma elevada prevalência de transtorno de ansiedade generalizada; depressão no passado, transtorno do pânico e agorafobia. Dos portadores de TCAP, $42,1 \%$ apresentavam depressão no momento da avaliação, contra apenas $13,8 \%$ do grupo sem TCAP, sendo essa associação significativa $(\mathrm{p}=0,012)$.

O ponto de corte utilizado na BES foi o escore maior que 17 , de acordo com recomendação dos autores que validaram a escala (20). A média dos escores obtida no grupo com TCAP foi $25,76( \pm$ 5,36 ), sendo verificado o transtorno em $56,7 \%$ dos entrevistados $(25,4 \%$ dos pacientes com TCAP moderado e $31,3 \%$ grave).

Quanto aos domínios da escala de qualidade de vida SF-36, comparados ao grupo sem TCAP, os portadores de compulsão apresentaram piores escores na maioria dos parâmetros, com exceção do domínio vitalidade (houve uma tendência para um pior funcionamento no grupo com TCAP, embora sem significância estatística). Esses resultados estão na tabela 3.

Arq Bras Endocrinol Metab vol 50 n 5 Outubro 2006 
Tabela 3. Avaliação dos domínios da escala SF-36 segundo a presença ou ausência de TCAP.

\begin{tabular}{lcccc}
\hline Domínios (média/DP) & Grupo com TCAP & Grupo sem TCAP & Grupo total & $p$ \\
\hline Capacidade funcional & $35,92( \pm 19,48)$ & $48,97( \pm 28,07)$ & $41,57( \pm 24,28)$ & $\mathrm{p}(1)=0,037^{*}$ \\
Limitação dos aspectos físicos & $21,05( \pm 28,79)$ & $44,83( \pm 44,51)$ & $31,34( \pm 38,02)$ & $\mathrm{p}(1)=0,015^{*}$ \\
Dor & $35,21( \pm 22,21)$ & $51,41( \pm 32,41)$ & $42,22( \pm 28,06)$ & $\mathrm{P}(1)=0,025^{*}$ \\
Estado geral de saúde & $42,26( \pm 25,06)$ & $56,00( \pm 22,78)$ & $48,21( \pm 24,88)$ & $\mathrm{P}(2)=0,024^{*}$ \\
Vitalidade & $36,05( \pm 19,04)$ & $46,38( \pm 24,45)$ & $40,52( \pm 21,99)$ & $\mathrm{p}(2)=0,056$ \\
Aspectos sociais & $53,95( \pm 29,51)$ & $75,43( \pm 24,43)$ & $63,25( \pm 28,27)$ & $\mathrm{P}(2)=0,002^{*}$ \\
Limitação dos aspectos emocionais & $36,84( \pm 41,58)$ & $58,62( \pm 43,33)$ & $46,27( \pm 43,41)$ & $\mathrm{p}(1)=0,040^{*}$ \\
Saúde mental & $51,05( \pm 21,28)$ & $67,17( \pm 21,44)$ & $58,03( \pm 22,66)$ & $\mathrm{p}(2)=0,003^{*}$ \\
\hline
\end{tabular}

( $^{*}$ ) Diferença significante ao nível de 5,0\%

(1) Através do teste t-Student com variâncias desiguais

(2) Através do teste t-Student com variâncias iguais

\section{DISCUSSÃO}

Nas últimas décadas, as pressões sociais por um corpo esbelto têm se intensificado e as pessoas com sobrepeso são freqüentemente avaliadas de forma depreciativa, sendo alvo de preconceito e discriminação nos locais de trabalho, relacionamentos sociais, assim como pelos profissionais de saúde, devido à sua aparência física.

A obesidade não é classificada como um transtorno psiquiátrico (22). Esta afirmação se faz necessária desde que, por muito tempo, a obesidade foi compreendida como uma manifestação somática de um conflito psicológico que, em determinados indivíduos com formação egóica inadequada, seria solucionado através da alimentação excessiva. Esta visão é ainda hoje compartilhada tanto pela população leiga como por uma boa parte dos profissionais de saúde. Esta postura não tem sido mais aceita, ao menos quando se fala de obesos da população geral.

Tem sido largamente pesquisado se os obesos têm maiores índices de transtornos psiquiátricos. Em estudos de amostras comunitárias, os indivíduos com obesidade geralmente não têm mais psicopatologia do que os com peso normal. Em contraste a isso, entre obesos que procuram tratamento têm sido encontrados índices significativamente elevados de depressão (23-25) e mais modestos de transtornos de ansiedade (incluindo agorafobia, fobia simples e transtorno de estresse pós-traumático), bulimia, tabagismo e transtorno de personalidade borderline $(23,24)$.

Essa discrepância entre amostras comunitárias e clínicas pode refletir a tendência dos indivíduos que sofrem de algum transtorno comórbido de serem mais comprometidos e, portanto, mais propensos a procurar tratamento. De qualquer forma, na medida em que lidamos com populações clínicas, o achado de maior depressão comórbida é relevante e deve ser considerado ao se avaliar pacientes que se apresentam para trata- mento de obesidade. No entanto, até agora parece não ser possível estabelecer uma relação de causa-efeito.

Esses achados foram verificados no presente estudo. Depressão atual foi encontrada em 29,9\% da amostra total e transtornos de ansiedade, como transtorno de ansiedade generalizada, transtorno do pânico e agorafobia, também foram muito prevalentes tanto no grupo com TCAP quanto no grupo sem TCAP. Onyike e cols. (26), utilizando dados do Third National Health and Nutrition Examination Survey, encontraram que a associação entre obesidade e depressão depende da gravidade da obesidade. Há um aumento da psicopatologia em pacientes gravemente obesos (obesidade grau III) $(17,18)$. Todos esses transtornos apresentaram-se em freqüência mais elevada que na população geral.

A associação entre excesso de peso corporal e transtornos psiquiátricos poderia ser explicada pelo seguimento de dietas ou por outras doenças, sendo de relevância o TCAP. Obesos com TCAP apresentam obesidade mais precoce que os não-compulsivos, iniciam mais cedo a preocupação com peso e dietas, apresentam maior flutuação de peso e passam mais tempo de suas vidas tentando emagrecer $(1,13)$. Tendem a engordar se comparados àqueles sem esta perturbação. Os níveis de psicopatologia exibidos estão associados ao número de episódios de compulsão alimentar que experimentam e não ao seu grau de obesidade (22). O TCAP está relacionado a sintomas psicopatológicos em geral (sobretudo depressão), a maior gravidade da obesidade e a prejuízo no funcionamento social e ocupacional $(6,10,13-16)$. Na nossa amostra observamos que os obesos portadores de TCAP tiveram maior número de tratamentos para emagrecer e apresentam mais depressão que os não-compulsivos.

Uma área de interesse crescente é a avaliação da qualidade de vida dos obesos. Recomenda-se o uso de escalas de avaliação validadas, visando estabelecer que variáveis podem interferir na qualidade de vida desses 
indivíduos. A Baros (27) tem sido amplamente utilizada na avaliação de obesos mórbidos submetidos à gastroplastia. No entanto, nas fases de avaliação e no préoperatório os estudos são ainda escassos. É sabido que comedores-compulsivos que procuram tratamento apresentam mais psicopatologia que os obesos nãocompulsivos. De acordo com Kolotkin e cols. (28), apenas três estudos avaliaram o impacto do TCAP na qualidade de vida e, em todos eles, foi verificada a existência de uma associação estreita. No primeiro, Marchesini e cols. (29) observaram a significativa associação entre TCAP e comprometimento na escala de qualidade de vida SF-36, particularmente nos domínios da saúde mental. Já Hsu e cols. (30) relataram que os obesos mórbidos portadores de TCAP apresentaram piores escores em três domínios da SF-36. Kolotkin e cols. (28) verificaram que essa associação é reduzida quando controladas as variáveis depressão e auto-estima. No entanto, apesar do controle das variáveis, De Zwaan e cols. observaram que os portadores de TCAP mantiveram piores escores em três das cinco sub-escalas da Weight on Quality of Life-Lite (IWQOL-Lite) (31), escala específica para avaliação da qualidade de vida em obesos, cuja validação para o português ainda não foi publicada. Talvez por ser uma escala específica é mais sensível para os problemas relacionados à obesidade.

Mais recentemente, Rieger e cols. (32) também observaram que obesos com TCAP apresentam um maior comprometimento nos aspectos psicossociais da escala de qualidade de vida em relação aos não-compulsivos, e enfatizaram que o TCAP foi mais associado ao comprometimento do que a obesidade sozinha. Ainda nessa mesma linha de investigação, Masheb e Grillo (33) verificaram que os comedores-compulsivos apresentavam um pior funcionamento $\mathrm{em}$ todos os domínios da SF-36, quando comparados com controles normais, bem como em alguns domínios, quando comparados a obesos que buscaram tratamento.

Um dos achados mais importantes da nossa pesquisa foi a observação de que os pacientes com TCAP apresentaram piores escores em todos os domínios da SF-36 quando comparados ao grupo sem TCAP, havendo, pois, uma concordância com a literatura $(29,30)$

O TCAP foi verificado em $56,7 \%$ dos entrevistados, freqüência maior dos 5 a $50 \%$ encontrados em obesos que buscam serviços especializados para tratamento da obesidade (1-4). Essas cifras elevadas nos levou a refletir sobre as suas possíveis causas. Ressaltamos que a nossa amostra foi constituída por pacientes pobres, que aguardam mais de dois anos entre a entra- da na lista de espera e a realização da cirurgia bariátri$\mathrm{ca}$, portadores de múltiplas tentativas frustradas para perder peso, e $42 \%$ preenchiam critérios para depressão no momento da entrevista. O estresse é um fator que pode levar ao aumento das compulsões alimentares (34). Durante situações estressantes, o cortisol é liberado estimulando a ingestão de alimentos e o aumento do peso - situação essa possível de acometer parte desses pacientes (34).

Uma outra hipótese levantada reside, sem dúvidas, em uma das limitações do nosso estudo. $\mathrm{O}$ diagnóstico de TCAP foi realizado apenas pelo preenchimento da BES. Embora seja um questionário autoaplicável, como a maioria dos pacientes tinha um baixo nível de escolaridade (57\% apenas o primeiro grau), terminou por ser preenchido pelas entrevistadoras, juntamente com os outros instrumentos. Alguns autores têm alertado que os instrumentos de autoavaliação podem superestimar a prevalência de TCAP (35). Greeno e cols. (36) afirmaram que a BES é útil na identificação de indivíduos sem CAP e também como ferramenta para rastrear pacientes com graves problemas de CAP. Lembram que como não se correlaciona aos critérios para o TCAP do DSM-IV, que seja seguido por uma entrevista clínica. Nessa mesma linha de raciocínio, Gladis e cols. (37) acentuam que a BES é uma boa medida para investigar as cognições relacionadas à alimentação e ao peso e para avaliar o sofrimento a elas associado. Altos escores poderiam ser mais um marcador da psicopatologia associada ao transtorno do que o próprio transtorno em si.

Dessa forma, todos os pacientes entrevistados e que apresentassem altos escores na BES deveriam ter sido reavaliados por um profissional experiente e treinado na aplicação desses instrumentos, além da inclusão dos critérios do DSM-IV para TCAP. Lembramos que utilizamos uma entrevista para o diagnóstico psiquiátrico, o MINI, mas que por ser uma entrevista breve só explora 17 transtornos do eixo I do DSM-IV, não abrangendo o TCAP. Uma outra limitação do presente trabalho foi o desenho de corte-transversal. Apesar de ter sido encontrada uma associação entre TCAP e piores escores na SF-36, o desenho não nos permite fazer inferências de relação causal. Sem dúvidas que estudos prospectivos se fazem necessários.

Concluímos que o grupo com TCAP apresenta maior número de tratamentos realizados com objetivo de perder peso, elevada prevalência de depressão maior no momento da avaliação, piores escores em todos os domínios da escala de qualidade de vida SF-36. Foi observada ainda uma alta prevalência de TCAP e de outros transtornos psiquiátricos. 
Ainda não há um consenso na literatura sobre os critérios para avaliação psiquiátrica nos candidatos às operações bariátricas. Cada equipe multidisciplinar parece usar seus próprios critérios. Transtornos psiquiátricos, especialmente do humor, ansiosos e psicóticos são comumente considerados contra-indicações para o procedimento. Contudo não há dados precisos nem fatores preditivos de bom ou mau prognóstico adequadamente estudados e/ou comprovados. Esta é uma área de interesse, não só do ponto de vista científico como também clínico (38).

Visto que os portadores de TCAP apresentam vasta psicopatologia, deve-se aprimorar a detecção desses distúrbios a fim de proporcionar-lhes o tratamento adequado. Os autores sugerem a avaliação psiquiátrica sistemática de todos os pacientes candidatos à cirurgia bariátrica, através de escalas de avaliação validadas, aplicadas pela equipe. Os pacientes que obtiverem altos escores nessas escalas devem ser reentrevistados por um psiquiatra experiente em transtornos alimentares.

\section{REFERÊNCIAS}

1. Spitzer RL, Yanovski S, Wadden T, Wing R, Marcus MD, Stunkard A, et al. Binge Eating Disorder: its further validation in a multisite study. Int J Eat Disord 1993; 13:13753.

2. Grilo CM. Binge eating disorder. In: Fairburn CG, Brownell KD (eds). Eating disorders and obesity: a comprehensive handbook. $2^{\text {nd }}$ ed. New York: Guilford Press, 2002. pp. 178-82.

3. de Zwaan M. Binge eating disorder and obesity. Int $\mathbf{J}$ Obes Relat Metab Disord 2001;25:S51-5.

4. Marchesini G, Natale S, Chierici S, Manini R, Besteghi L, Di Domizio $S$, et al. Effects of cognitive-behavioural therapy on health-related quality of life in obese subjects with and without binge eating disorder. Int J Obes Relat Metab Disord 2002;26:1261-7.

5. Appolinário JC, Coutinho W, Povoa LC. O transtorno do comer compulsivo: revisão da literatura. J Bras Psiquiatr 1995;44(1):S38-45.

6. Coutinho W. Estudo da compulsão alimentar periódica em pacientes que procuram tratamento médico para emagrecer (Tese). São Paulo: Universidade Federal de São Paulo; 2000.

7. Borges MB. Estudo do transtorno da compulsão alimentar periódica em população de obesos e sua associação com depressão e alexitimia (Dissertação). São Paulo: Universidade Federal de São Paulo, 1998.

8. Wadden TA, Sarwer DB, Womble LG, Foster GD, McGuckin BG, Schimmel A. Psychosocial aspects of obesity and obesity surgery. Sur Clin North Am 2001;81:1001-24.
9. Smith DE, Marcus MD, Lewis CE, Fitzgibbon M, Schreiner P. Prevalence of binge eating disorder, obesity, and depression in a biracial cohort of young adults. Ann Behav Med 1998:20:227-32.

10. Napolitano MA, Head S, Babyak MA, Blumenthal JA. Binge eating disorder and night eating syndrome: psychological and behavioral characteristics. Int J Eat Disord 2001;30:193-203.

11. Stunkard AJ, Allison KC. Two forms of disordered eating in obesity: binge eating and night eating. Int $\mathbf{J}$ Obes 2003;27:1-12

12. American Psychiatric Association. Diagnostic and statistical manual of mental disorders. $4^{\text {th }}$ ed. (DSM-IV-TR), Washington: American Psychiatric Publishing, 1994.

13. Coutinho W. Comer compulsivo. In: Obesidade. São Paulo: Lemos Editorial, 1998. pp. 209-16.

14. Appolinario JC. Obesidade e psicopatologia. In: Obesidade. São Paulo: Lemos Editorial, 1998. pp. 217-23.

15. Goldfein JA, Walsh BT, La Chaussee JL, Kissileff HR, Devlin MJ. Eating behavior in binge eating disorder. Int J Eat Disord 1993; 14:427-31.

16. Azevedo AP, Santos CC, Fonseca DC. Transtorno da compulsão alimentar periódica. Rev Psiquiatr Clín 2004;31(4):70-2.

17. Adami GF, Gandolfo P, Bauer B, Scopinaro N. Binge eating in massively obese patients undergoing bariatric surgery. Int J Eat Disord 1995; 17:45-50.

18. Fandino J, Benchimol AK, Coutinho WF, et al. Cirurgia bariátrica: aspectos clínico-cirúrgicos e psiquiátricos. Rev Psiquiatr RS 2004;26(1):47-51.

19. Amorim P. Mini International Neuropsychiatric Interview (MINI): validação de entrevista breve para diagnóstico de transtornos mentais. Rev Bras Psiquiatr 2000;22 (3): 106-15.

20. Freitas S, Lopes CS, Coutinho W, Appolinario JC. Tradução e adaptação para o português da Escala de Compulsão Alimentar Periódica. Rev Bras Psiquiatr 2001:23(4):215-20.

21. Ciconelli RM. Tradução para o português e validação do questionário genérico de avaliação de qualidade de vida "Medical Outcomes Study 36-Short item-Form Health Survey" (Tese). Universidade Federal de São Paulo, SP, 1997. p. 95.

22. Dobrow IJ, Kamenetz C, Devlin MJ. Aspectos psiquiátricos da obesidade. Rev Bras Psiquiatr 2002;24(3):63-7.

23. Goldsmith SJ, Anger-Friedfeld K, Beren S, Boeck M, Aronne L. Psychiatric illness in patients presenting for obesity treatment. Int J Eat Dis 1992; 12:63-71.

24. Black DW, Goldstein RB, Mason EE. Prevalence of mental disorder in 88 morbidly obese bariatric clinic patients. Am J Psychiatry 1992;149:227-34.

25. Maddi SR, Khoshaba DM, Persico M, Bleecker F, VanArsdall G. Psychosocial correlates of psychopathology in a national sample of the morbidly obese. Obes Surg 1997:7:397-404.

26. Onyike CU, Crum RM, Lee HB, Lyketsos CG, Eaton WW. Is obesity associated with major depression? Results from the Third National Health and Nutrition Examination Survey. Am J Epidemiol 2003; 158(12):1139-47. 
27. Wolf AM, Falcone AR, Kortner B, Kuhlmann HW. BAROS: an effective system to evaluate the results of patients after bariatric surgery. Obes Surg 2000; 10(5):445-50.

28. Kolotkin RL, Westman EC, Østbye O, Crosby RD, Eisenson HJ, Binks M. Does Binge Eating Disorder impact weightrelated quality of life? Obes Res 2004; 12:999-1005.

29. Marchesini G, Solaroli E, Baraldi L, Natale S, Migliorini S, Visani $F$, et al. Health-related quality of life in obesity: the role of eating behaviour. Diabetes Nutr Metab 2000;13:156-64.

30. Hsu LK, Mulliken B, McDonagh B, Krupa Das S, Rand W, Fairburn CG, et al. Binge eating disorder in extreme obesity. Int J Obes 2002;26:1398-403.

31. De Zwaan M, Mitchell JE, Howell LM, Monson N, SwanKremeier L, Roerig JL, et al. Two measures of health-related quality of life in morbid obesity. Obes Res 2002; 10(11):1143-51.

32. Rieger E, Wilfley DE, Stein RI, Marino V, Crow SJ. A comparison of quality of life in obese individuals with and without binge eating disorder. Int J Eat Disord 2005;37(3):234-40.

33. Masheb RM, Grilo CM. Quality of life in patients with binge eating disorder. Eat Weight Disord 2004;9(3):194-9.
34. Gluck ME, Geliebter A, Lorence M. Cortisol stress response is positively correlated with central obesity in obese women with binge eating disorder (BED) before and after cognitive-behavioral treatment. Ann NY Acad Sci 2004; 1032:202-7.

35. Stunkard AJ, Allison KC. Binge eating disorder: disorder or marker? Int J Eat Disord 2003;34:S107-16.

36. Greeno CG, Marcus MD, Wing RR. Diagnosis of binge eating disorder: discrepancies between a questionnaire and clinical interview. Int J Eat Dis 1995;17(2):153-60.

37. Gladis MM, Wadden TA, Foster GD, Vogt RA, Wingate BL. A comparison of two approaches to the assessment of binge eating in obesity. Int J Eat Dis 1998;23(1):17-26.

38. Segal A, Fandino J. Indicações e contra-indicações para realização das operações bariátricas. Rev Bras Psiquiatr 2002;24(3):68-72.

\section{Endereço para correspondência:}

Kátia Petribu

Rua Apipucos 235, apto. 602

52071-000 Recife, PE

E-mail: katia@elogica.com.br 